\title{
Primed Facilitation of Homosynaptic Long-Term Depression and Depotentiation in Rat Hippocampus
}

\author{
Lorne L. Holland and John J. Wagner \\ Department of Pharmaceutical Sciences, College of Pharmacy, North Dakota State University, Fargo, \\ North Dakota 58105
}

\begin{abstract}
Previous studies have demonstrated that prior synaptic activity can influence the subsequent induction of synaptic plasticity in the brain. Such temporal modulation of synaptic plasticity has been called "metaplasticity." In this report, we describe the facilitatory effects of high-frequency stimulation on the induction of homosynaptic long-term depression (LTD) in the CA1 region of the rat hippocampus. The LTD induced by lowfrequency stimulation $(1 \mathrm{~Hz})$ protocols was found to be homosynaptic and NMDA receptor-dependent. The facilitatory effects of the high-frequency stimulation-induced priming event itself were found to be NMDA receptor-independent and to
\end{abstract}

have a duration of at least 90 min. The effects of priming also were heterosynaptic, because the induction of synaptic plasticity by low-frequency stimulation was enhanced at an unprimed synaptic pathway after the priming of an independent pathway. In addition to enhancing LTD, priming also enhanced the reversal of long-term potentiation elicited by a $5 \mathrm{~Hz}$ depotentiation protocol. Our results provide examples of how metaplasticity may play a key role in the ongoing modulation of the induction and stabilization of alterations in synaptic strength.

Key words: synaptic plasticity; LTP; LTD; depotentiation; priming; metaplasticity; hippocampus
Activity-dependent modulation of synaptic plasticity is an important component in the current understanding of the cellular mechanisms underlying learning and memory functions of the brain (Abraham, 1996; Abraham and Bear, 1996). The term metaplasticity has been suggested to encompass these phenomena, which generally involve some previous synaptic activity that results in a change in the capabilities of the synapse to undergo subsequent plasticity (Bear, 1995). Such temporal plasticity of synaptic plasticity can last for minutes to hours and is present in the absence of any discernible effects on baseline levels of synaptic transmission. Metaplasticity may be particularly relevant in the study of long-term depression (LTD), because there have been significant discrepancies in both past and recent literature concerning the occurrence of homosynaptic LTD induced by lowfrequency stimulation (LFS; $1 \mathrm{~Hz}$ ) in the hippocampal formation of adult rats (see Wagner and Alger, 1996; Heynen et al., 1996; Staubli and Scafidi, 1997). One consistent finding in this literature is that the occurrence of previous synaptic activity significantly enhances the likelihood of subsequently observing LTD (Christie and Abraham, 1992; Wexler and Stanton, 1993; Wagner and Alger, 1995). Because LFS-induced LTD can be observed under certain conditions, it would seem that this form of LTD is deserving of continued attention as a potentially important plasticity component in the processing of information by neuronal networks.

In this report, we have examined the modulatory effects of a high-frequency stimulation (HFS) priming protocol on the subsequent induction of LTD and depotentiation in the CA1 region of rat hippocampal slices. The properties of this LTD expressed

\footnotetext{
Received Sept. 11, 1997; revised Nov. 13, 1997; accepted Nov. 17, 1997.

This work was supported by National Institute on Drug Abuse Grant DA09603 to J.W. We thank Dr. Brad Alger and colleagues for helpful comments on an earlier version of this manuscript.

Correspondence should be addressed to Dr. John J. Wagner, College of Pharmacy, North Dakota State University, 123 Sudro Hall, Fargo, ND 58105.

Copyright (C) 1998 Society for Neuroscience $0270-6474 / 98 / 180887-08 \$ 05.00 / 0$
}

after a priming event (i.e., primed LTD) were compared with those of the typical LFS-induced homosynaptic LTD. In addition, the priming phenomenon itself was characterized with respect to its duration and its pathway specificity. A recent study has suggested that after the induction of protein synthesis-dependent LTP (late LTP), a temporal window exists that allows stabilization of heterosynaptic early LTP such that it too becomes late LTP (Frey and Morris, 1997). Although this would allow for the long-term storage of associated events, it would also potentially result in the inappropriate storage of extraneous information. Because our priming protocol is comparable with those used to elicit late LTP, we tested the hypothesis that the effects of late LTP induction at one set of synapses might also impact the efficacy of LTD or depotentiation protocols at another, independent set of synapses. Indeed, we find that priming does have heterosynaptic effects, resulting in an enhancement of both LTD and depotentiation in the heterosynaptic pathway. We propose that the primed facilitation of LTD and/or depotentiation after the induction of late LTP may represent examples of "safeguard" mechanisms that act to prevent or decrease the storage of extraneous information.

\section{MATERIALS AND METHODS}

Extracellular electrophysiology. Freshly prepared transverse hippocampal slices $(500 \mu \mathrm{m})$ were obtained from mature (40-80-d-old) Sprague Dawley rats that had been anesthetized (halothane) before decapitation. The CA3 region was removed surgically immediately after slice dissection. Slices were then submerged in a recording chamber and perfused continuously with saline saturated with $95 \% \mathrm{O}_{2} / 5 \% \mathrm{CO}_{2}$ at $\sim 1 \mathrm{ml} / \mathrm{min}$. The recording chamber and perfusion saline were then warmed to $\sim 29^{\circ} \mathrm{C}$ for the duration of the experiment. The slices were incubated for at least $1 \mathrm{hr}$ in the chamber before an experiment was begun. The saline contained (in mM): $\mathrm{NaCl} 120, \mathrm{KCl} 3, \mathrm{MgCl}_{2} 1.5, \mathrm{NaH}_{2} \mathrm{PO}_{4} 1, \mathrm{CaCl}_{2} 2.5$, $\mathrm{NaHCO}_{3} 26$, and glucose 10. Extracellular recording electrodes $(1-2 \mu \mathrm{m}$ tip) were filled with $200 \mathrm{~mm} \mathrm{NaCl}$ and placed in the stratum radiatum of CA1. The field EPSP population responses were evoked with a bipolar stimulating electrode (Kopf Instruments, Tujunga, CA) placed on either the $\mathrm{CA} 3$ or the subicular side of the recording electrode in the stratum 
radiatum. Stimulation parameters consisted of single square waves of 40-100 $\mu \mathrm{A}$ of $300 \mu \mathrm{sec}$ duration. Data were digitized at $10 \mathrm{kHz}$ and analyzed with pCLAMP 6 software (Axon Instruments, Foster City, CA). The initial slope of the population EPSP was measured by fitting a straight line to the first millisecond of the EPSP immediately after the fiber volley artifact.

Stimulus-response curves were performed at the beginning of each experiment. Pulses of an intensity that gave $40-60 \%$ of the maximum response were given at a frequency of $0.05 \mathrm{~Hz}$ for the remainder of the experiment. All stimulation protocols were performed at the test pulse intensity, and when two synaptic pathways were monitored, their independence was evaluated as described previously (Wagner and Alger, 1995). The $1 \mathrm{~Hz}$ LFS protocol consisted of a 10 min period of $1 \mathrm{~Hz}$ stimulation that was repeated after a $10 \mathrm{~min}$ interval. The $5 \mathrm{~Hz} \mathrm{LFS}$ protocol consisted of a $1 \mathrm{~min}$ period of $5 \mathrm{~Hz}$ stimulation that was repeated after a $2.5 \mathrm{~min}$ interval. For clarity, the responses during LFS are not illustrated in the figures. Priming (and/or late LTP induction) was induced using two sets of three HFS $(100 \mathrm{~Hz} / 1 \mathrm{sec})$ trains given at an interval of $20 \mathrm{sec}$, with $15 \mathrm{~min}$ between sets. Early LTP was induced by using a single $100 \mathrm{~Hz} / 1 \mathrm{sec}$ train. This single-tetanus protocol produced LTP (defined as a $\geq 20 \%$ increase in field EPSP slope) lasting $<3 \mathrm{hr}$ in a group of control slices $(n=4)$.

Quantification of synaptic plasticity. LTD was quantified 25-30 min after the completion of the LFS protocol by averaging the EPSP slopes from 15 consecutive responses at baseline frequency and dividing this value by the average of the 15 EPSP slopes from $5 \mathrm{~min}$ before beginning LFS. Depotentiation was quantified by defining the amount of potentiation remaining $15 \mathrm{~min}$ after the last HFS as $100 \%$, and then determining the percentage of potentiation remaining after LFS, as was performed for LTD. Control values for depotentiation (i.e., rundown of LTP) were obtained using control groups of LTP experiments that did not undergo LFS. Unless otherwise noted, the $n$ values reported represent slices taken from different animals for a given experimental group (e.g., $n=6$ is six slices obtained from six different animals).

\section{RESULTS}

\section{Primed LTD is similar to typical LFS-induced LTD}

The field EPSP was monitored in the stratum radiatum of the CA1 region in response to stimulation of the Schaffer collaterals. An LFS protocol consisting of two episodes of $1 \mathrm{~Hz}$ stimulation/ 600 pulses separated by a 10 min interval was used in the attempt to elicit LTD. As shown in Figure $1 A, 1 \mathrm{~Hz}$ stimulation had little effect on the baseline response in slices obtained from 40- to 80 -d-old rats $(92 \pm 3 \%, n=6$ slices from four animals). In contrast, a significantly enhanced depression was observed (77 \pm $2 \%, n=6$ ) when an HFS priming protocol preceded the LFS episodes $($ at $* *$, the tetani were administered in the presence of DL-APV, $100 \mu \mathrm{M}$, to prevent LTP) (Fig. $1 B$ ).

This primed LTD is dependent on the activation of NMDA receptors during the LFS episodes, because LFS performed in the continued presence of APV results in no significant depression from the baseline response $(98 \pm 4 \%, n=6$ slices from five animals) (Fig. 1C). To determine the pathway specificity of primed LTD, field EPSPs from two independent pathways were monitored during the experiments illustrated in Figure $1 B$ (control pathway not shown). The control pathway was significantly less affected by the LFS of the primed pathway $(89 \pm 3 \%, n=6$; $p<0.01$, paired $t$ test), indicating that primed LTD has a homosynaptic component. These findings are in agreement with other reports describing the properties of LFS-induced LTD (Dudek and Bear, 1992; Mulkey and Malenka, 1992).

\section{Duration of the priming effect}

The results of Figure $1 B$ establish that our priming protocol has effects lasting for at least the $30 \mathrm{~min}$ allowed for completing the APV washout. We then extended the time between the priming protocol and LFS episodes to determine the duration of the priming effect. As illustrated in Figure $2 A$, significant facilitation of LTD still could be elicited 90 min after priming $(82 \pm 3 \%, n=$ 4) when compared with temporally matched control (i.e., LFS without priming) experiments $(92 \pm 1 \%, n=4)$; however, the effects of LFS at $150 \mathrm{~min}$ after priming were not significantly different from its control group $(85 \pm 3 \%$ vs $89 \pm 4 \%$, respectively; $n=4$ for each). Another way of considering the data is illustrated in Figure $2 B$, in which the degree of facilitation (primed LTD magnitude/control LTD magnitude) is plotted versus the time after the priming protocol. These results demonstrate that the priming effect lasts at least 1.5 and $<2.5 \mathrm{hr}$.

\section{Primed facilitation of LTD is heterosynaptic}

The results described in Figure 1 suggest that priming facilitates the induction of an otherwise typical form of LTD elicited by LFS (i.e., NMDA receptor-dependent and homosynaptic); however, the characteristics of the priming phenomenon itself are a separate issue. As demonstrated previously (Wagner and Alger, 1995), and shown in Figure 1, this form of priming can be accomplished in the presence of APV, indicating that NMDA receptor activation is not required for priming. We also investigated the pathway specificity of priming itself by attempting to elicit LTD in an unprimed pathway $30 \mathrm{~min}$ after the priming of an independent pathway. The resulting LFS-induced depression in an unprimed pathway $(85 \pm 5 \%, n=8)$ (Fig. $3 B$ ) did not significantly differ from any of the conditions illustrated in Figure 1. This group result appeared to be attributable to the failure of priming to occur in some cases, because five experiments demonstrated obvious depression $(77 \pm 2 \%)$, and three experiments resulted in no depression $(100 \pm 3 \%)$. The underlying cause of the apparent failures to prime in the presence of APV was not investigated extensively, although we did note that the magnitude of the transient potentiation present after the HFS protocol was nearly twofold greater in the group that subsequently exhibited depression compared with the group that did not (data not shown). These results suggested to us that under certain conditions, primed facilitation of LTD indeed could be heterosynaptic.

Under physiologic conditions, NMDA receptors would be activated during the priming protocol; thus, we then tested the pathway specificity of the priming protocol in the absence of APV. In this situation, the priming protocol has consistent, significant heterosynaptic effects on LTD magnitude, as shown in Figure 3, $A$ and $B$. LFS of the unpotentiated pathway was given 30 min after the induction of LTP elicited by using the HFS priming protocol in an independent pathway. LTD in the unpotentiated, unprimed pathway $(81 \pm 2 \%, n=7)$ was significantly different from both the control and APV groups shown in Figure 1, $A$ and $C(p<0.05$, ANOVA followed by Student-Newman-Keuls post $h o c$ tests) and not significantly different from that seen in the primed pathway (Fig. $1 B$ ). In addition, the depression retains its pathway specificity, because the potentiated, primed pathway (Fig. 3A, solid squares) is not significantly affected $(92 \pm 2 \%, n=$ 7) after LFS of the other pathway (Fig. $3 A$, open squares) when compared with control LTP experiments monitored over the same amount of time (data not shown).

\section{Priming results in heterosynaptic facilitation of depotentiation}

One consequence of heterosynaptic priming would be that subsequent plasticity in independent pathways could be affected, either positively or negatively, depending on the type of plasticity induction event. The effects of a $5 \mathrm{~Hz}$ depotentiation protocol were tested on the maintenance of single-tetanus LTP (thought to 
A

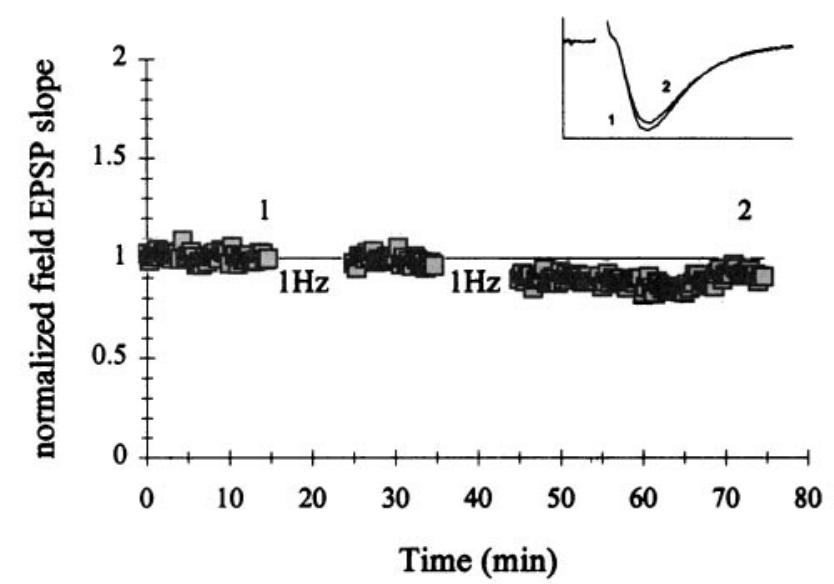

C

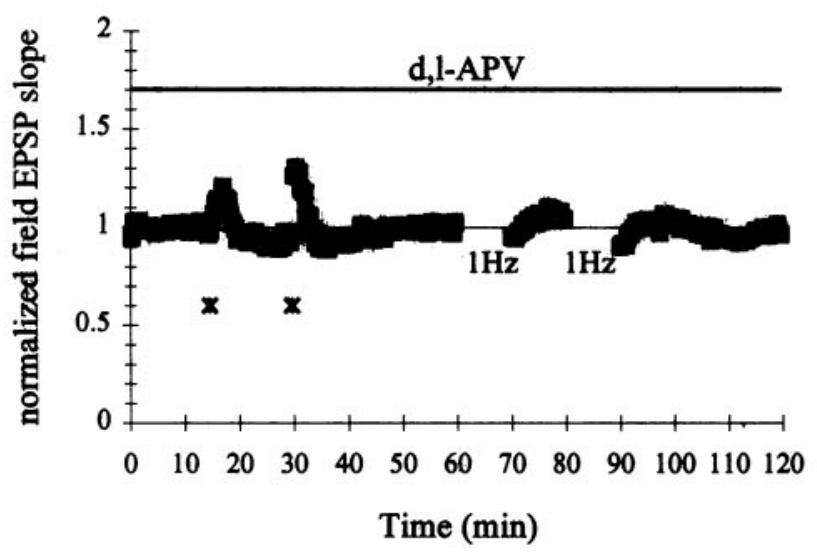

B

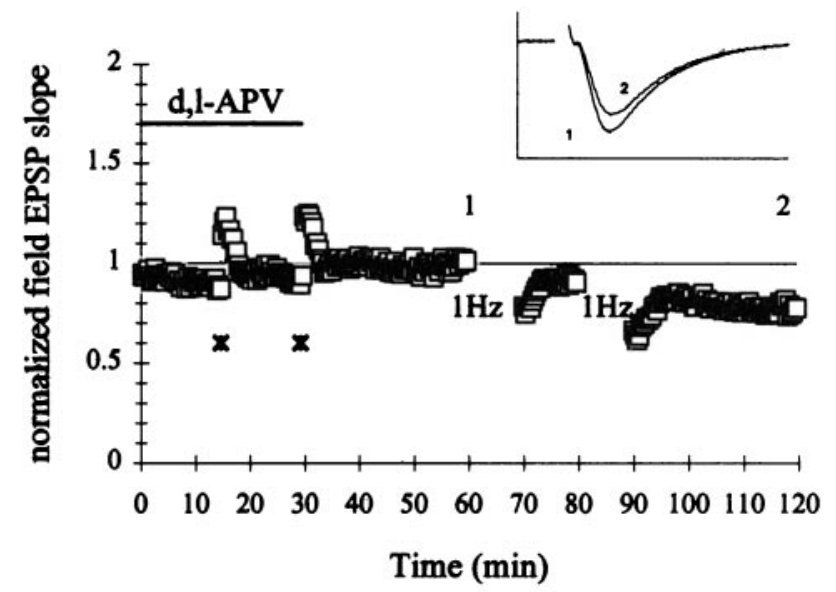

D

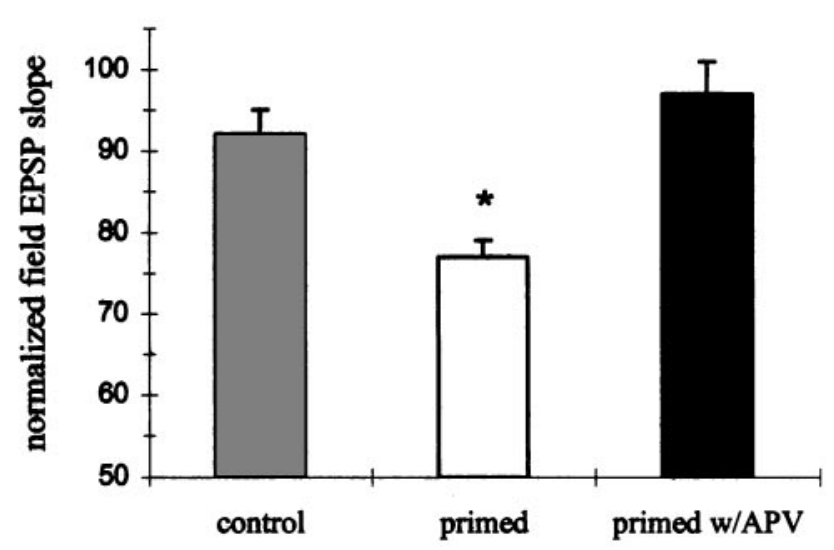

Figure 1. Priming facilitates the induction of NMDA receptor-dependent LTD. $A-C$, Summary plots $(n=6$ each) of normalized field EPSP measurements recorded every $20 \mathrm{sec}$ from the stratum radiatum layer of the CA1 region in hippocampal slices obtained from 6- to 11-week-old rats. The data are normalized to the 5 min period immediately preceding the first LFS episode and the period from 25 to 30 min after the second LFS was used to evaluate LTD magnitude. Insets are the averages of fifteen field potential sweeps (horizontal bar is $30 \mathrm{msec}$ ) from representative experiments at the indicated time points (vertical bar is $1.5 \mathrm{mV})$. A, Two LFS episodes $(600$ pulses $/ 1 \mathrm{~Hz}$ ) resulted in a modest, insignificant depression of the baseline response in naïve slices. $B$, The same LFS was effective in causing LTD when preceded by a priming protocol consisting of two HFS episodes (each consisting of $100 \mathrm{~Hz} / 1 \mathrm{sec} \times 3$ at $20 \mathrm{sec}$ intervals, as indicated by the asterisk) performed in the presence of DL-APV (100 $\mu \mathrm{M})$. $C$, The effects of LFS after priming were prevented in the continued presence of DL-APV $(100 \mu \mathrm{M})$. D, Summary quantitation from the above experiments. Error bars indicate the mean \pm SEM from six slices. The asterisk indicates a significant difference compared with the other conditions $(p<0.01$, ANOVA followed by Student-Newman-Keuls post hoc tests).

result in early LTP; see Materials and Methods) by comparing the magnitude of the potentiation remaining 30 min after depotentiation with the average potentiation measured at 10-15 min after HFS (Fig. 4). Consistent with previous reports, $5 \mathrm{~Hz}$ stimulation is largely ineffective at reversing LTP by $15 \mathrm{~min}$ after LTP induction (Fujii et al., 1991; Staubli and Chun, 1996). The fraction of potentiation remaining $30 \mathrm{~min}$ after two $5 \mathrm{~Hz}$ episodes $(1 \mathrm{~min}$ each, separated by $2.5 \mathrm{~min}$ ) was not significantly different from that seen in a control group (Fig. $4 \mathrm{~A}$, solid squares) followed over the same amount of time $(69 \pm 6 \%, n=6$, vs $79 \pm 6 \%, n=7$, respectively). In contrast, two $5 \mathrm{~Hz}$ episodes resulted in significant depotentiation relative to the time-matched control group (Fig. 4B, solid squares) when an independent pathway (**; otherwise not illustrated in the graph) was primed $30 \mathrm{~min}$ before LTP induction ( $49 \pm 4 \%, n=5$, vs $77 \pm 5 \%, n=5$, respectively).
There was no significant difference in the magnitude of potentiation induced among any of the four single-tetanus potentiation groups (data not shown). These results demonstrate that a significant facilitation of $5 \mathrm{~Hz}$ depotentiation of one input can occur after a priming event in another, separate input (Fig. 4D).

\section{The effect of $5 \mathrm{~Hz}$ depotentiation depends on the particular form of potentiation being reversed}

Our priming protocol consists of multiple $100 \mathrm{~Hz}$ trains and in the absence of APV is similar to an LTP induction protocol shown previously to result in late LTP, a protein synthesis-dependent, long-lasting form of LTP (Frey et al., 1993). Because of the intense nature of late LTP induction, we hypothesized that this type of potentiation might be more resistant to the depotentiating effects of $5 \mathrm{~Hz}$ stimulation than the early LTP induction protocol 
A

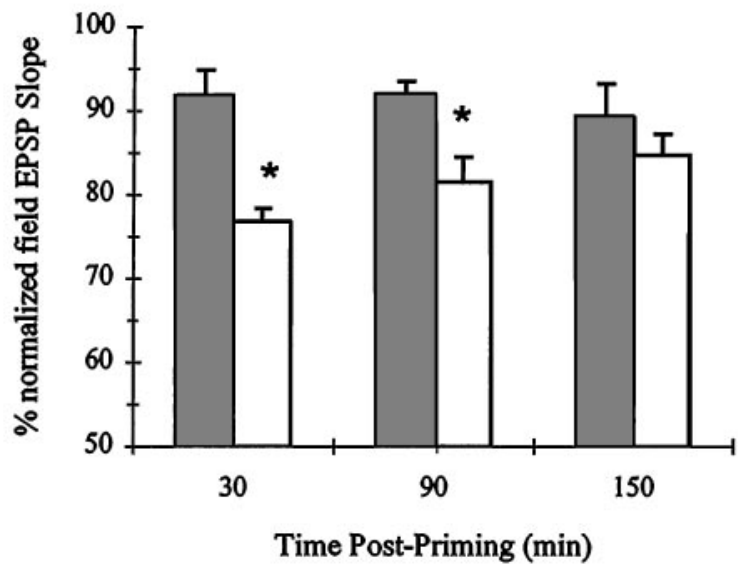

B

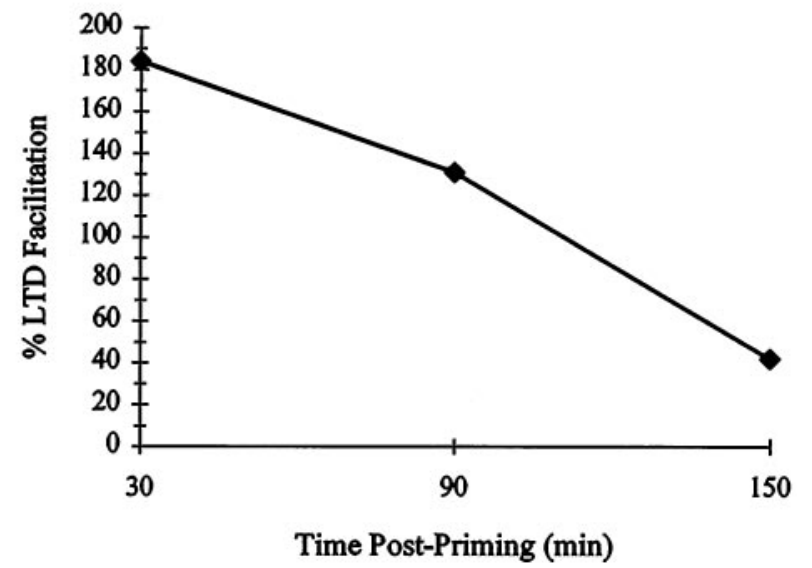

Figure 2. The facilitatory effects of priming on LTD last between 1.5 and $2.5 \mathrm{hr}$. $A$, Summary quantitation for control and primed LTD experiments performed as described in Figure 1, at the indicated priming-LFS intervals (open bars). Error bars indicate the mean \pm SEM from six, four, and four slices at 30, 90, and 150 min, respectively, for both control and primed groups. Asterisks Indicate a significant difference when compared with the corresponding temporal control group (unprimed, shaded bars) LFS group ( $p<0.01$, unpaired $t$ test). $B$, Using the data from Figure $2 A$, the relative increase in LTD magnitude at the indicated time points after priming is plotted (primed LTD magnitude/control LTD magnitude). For example, a doubling of LTD magnitude would be plotted as a $100 \%$ facilitation.

\section{A}

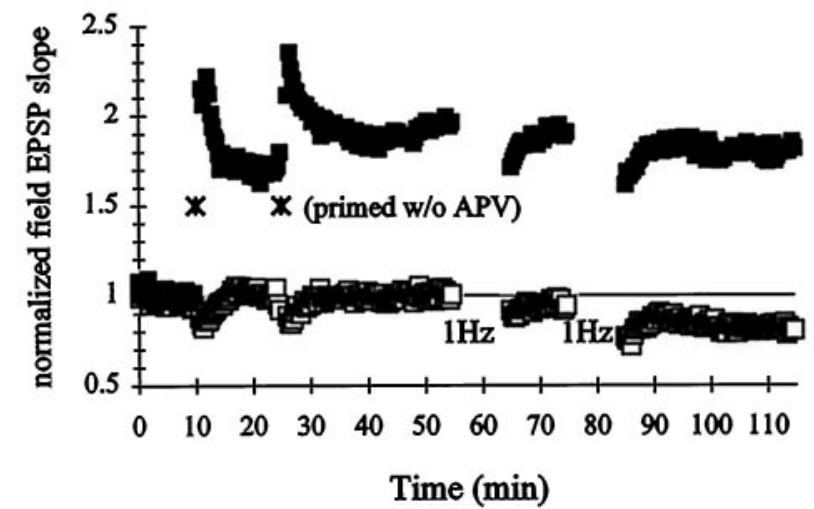

B

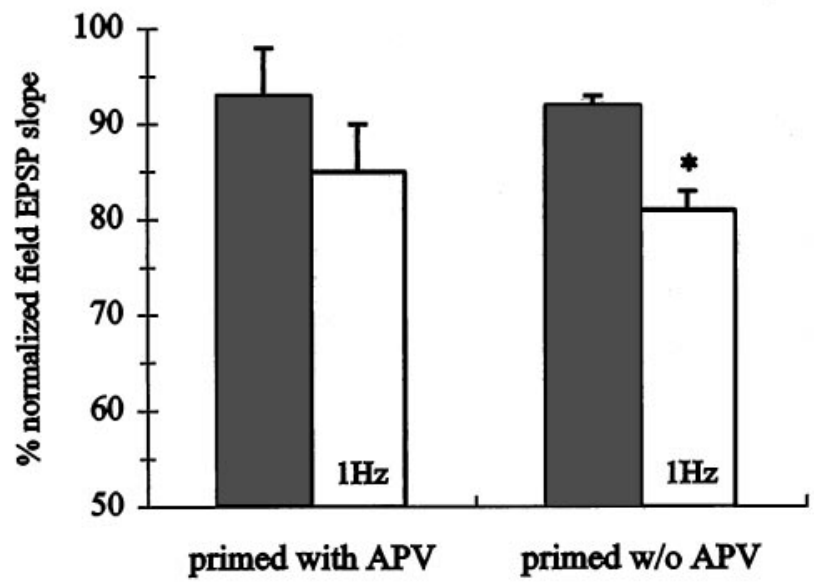

Figure 3. The effects of priming are expressed heterosynaptically. As in Figure 1, the data are normalized to the 5 min period immediately preceding the first LFS episode, and the period from 25 to $30 \mathrm{~min}$ after the second LFS was used to evaluate LTD magnitude. $A$, A summary plot $(n=7)$ of normalized field EPSP measurements from two independent pathways. Priming of one pathway (asterisks) was performed as in Figure 1, except in the absence of DL-APV, resulting in LTP of the primed pathway (solid squares). At 30 min after priming, LFS of the unprimed pathway (1 Hz) was performed, resulting in homosynaptic LTD (open squares). B. Summary quantitation from the experiment above (right set of of bars) and from a similar experiment (data not shown), in which DL-APV was present during the priming protocol (left set of bars, $n=8$ ). The open bars illustrate the effects of LFS performed in one pathway when the second pathway had been primed previously (i.e., heterosynaptic priming). The solid bars illustrate the data from the primed pathways (which did not experience LFS). Error bars indicate the mean \pm SEM, and asterisks indicate a significant depression compared with both the other pathway $(p<0.01, t$ test) and when compared with the data for Figure $1 A$ and $C$ ( $p<0.05$, ANOVA followed by Student-Newman-Keuls post hoc tests).

described above. Consistent with this prediction, the amount of potentiation remaining in the late LTP-induced pathway $30 \mathrm{~min}$ after a $5 \mathrm{~Hz}$ episode $(81 \pm 7 \%, n=6)$ was not significantly different than the decline seen in a control group followed over the same amount of time $(83 \pm 5 \%, n=10)$ (Fig. $5, A, C)$.

The above result could imply that a pathway experiencing a strong LTP induction protocol is in a consolidated state because of its lack of significant depotentiation by the $5 \mathrm{~Hz}$ episodes. However, consistent with our previous results using one episode of 900 pulses at $1 \mathrm{~Hz}$ (Wagner and Alger, 1995), we find that two 600 pulse episodes at $1 \mathrm{~Hz}$ significantly reversed the potentiation induced by multiple-tetanus trains (Fig. $5 B$ ) when compared with a temporally matched control group $(37+4 \%, n=4$, vs $72+5 \%$, $n=8$, respectively). There was no significant difference in the 
A

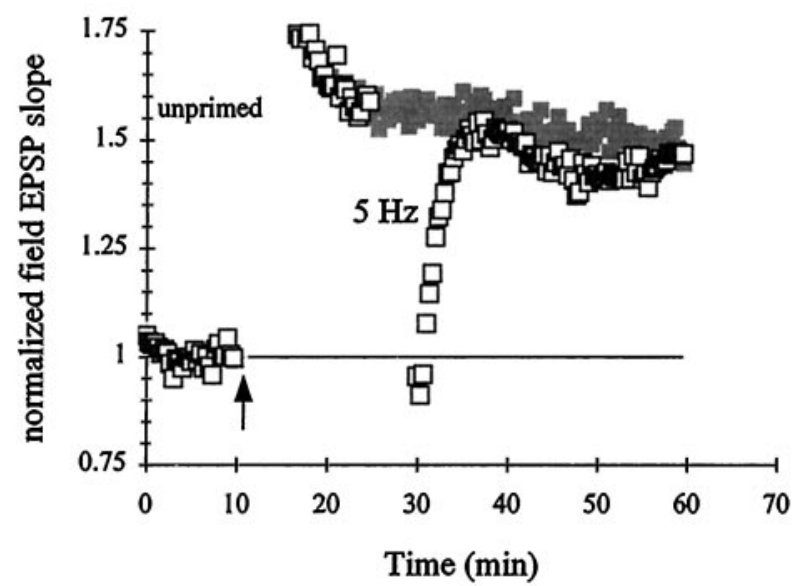

C

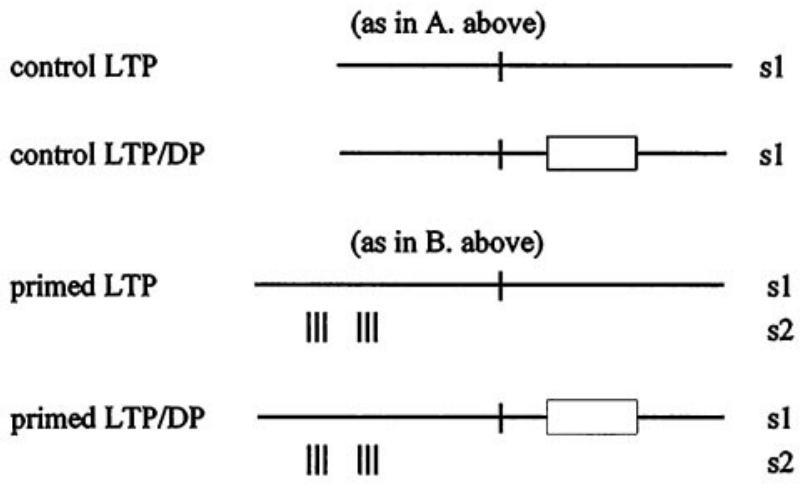

B

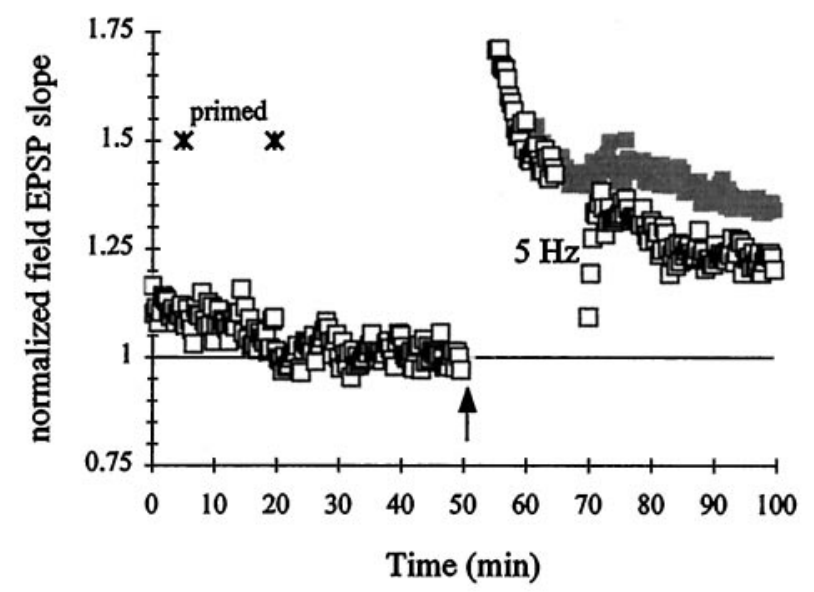

D

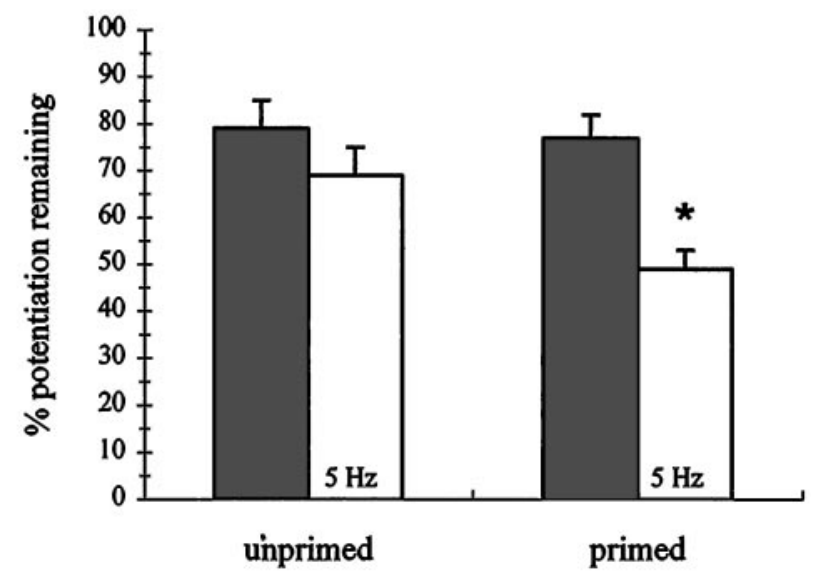

Figure 4. Heterosynaptic priming facilitates $5 \mathrm{~Hz}$ depotentiation. $A$, A summary plot $(n=6)$ of the LTP produced in one pathway after a single tetanus of $100 \mathrm{~Hz} / 1 \mathrm{sec}$ (arrow). At 15 min after HFS, a $5 \mathrm{~Hz}$ depotentiation protocol consisting of two 1 min episodes separated by 2.5 min was performed (open squares). Superimposed is the time course of a control, single-tetanus experimental group $(n=7)$, which did not experience $5 \mathrm{~Hz}$ depotentiation (solid squares). These control data were renormalized to the $5 \mathrm{~min}$ period immediately preceding $5 \mathrm{~Hz}$ stimulation to account for a small difference in the magnitude of LTP in the two groups. $B$, As described in $A$, except the single-tetanus HFS was preceded by multiple-tetanus priming (as described for Fig. $3 A$ ) of an independent pathway (asterisks, pathway not shown). A summary plot $(n=5)$ of the LTP produced in one pathway after single-tetanus HFS (arrow). At $15 \mathrm{~min}$ after HFS, the $5 \mathrm{~Hz}$ depotentiation protocol was performed (open squares). Superimposed is the time course of a control, single-tetanus group $(n=5)$, which also had experienced heterosynaptic priming, but not $5 \mathrm{~Hz}$ depotentiation (solid squares). As in $A$, these control data were renormalized to the $5 \mathrm{~min}$ period immediately preceding $5 \mathrm{~Hz}$ stimulation to account for a small difference in the magnitude of LTP in the two groups. $C$, Schematic representations of the experiments performed in $A$ and $B$. Vertical lines denote $100 \mathrm{~Hz} / 1$ sec events. The boxes represent the $5 \mathrm{~Hz}$ depotentiation $(D P)$ protocol. $s 1$ And $s 2$ indicate independent synaptic pathways for the heterosynaptic priming performed in $B$. $D$, Summary quantitation from the experiments above. Error bars are the mean \pm SEM, and the asterisk indicates a significant difference compared with the control LTP group ( $p<0.01$, unpaired $t$ test).

magnitude of potentiation induced between any of the multipletetanus potentiation groups (data not shown). Thus, consolidation of the potentiation after the multiple-tetanus induction had not occurred, because a $1 \mathrm{~Hz}$ protocol still was effective in significantly depotentiating the LTP of the primed pathway (Fig. $5 C$ ). This suggests that the reversal of late LTP is sensitive to the particular depotentiation protocol being used.

\section{DISCUSSION}

Alterations in the strengths of a synaptic connections have long been proposed to underlie the cellular mechanisms that are involved in the acts of learning and memory by the brain (Hebb,
1949). Within this general hypothesis, the potential for bidirectional changes in the strength of synaptic connections is crucial for preserving the capability for change at a given synapse and for providing the maximum flexibility and capacity of the system as a whole (Sejnowski, 1977; Bienenstock et al., 1982). Thus, understanding the processes governing the ability to both increase and decrease synaptic strength is central to determining how learning and memory occur in the nervous system. In this study, we have found that primed LTD in the CA1 region of hippocampal slices obtained from mature rats (6-11 weeks) exhibits the same fundamental properties as those of LFS-induced LTD in the CA1 
A

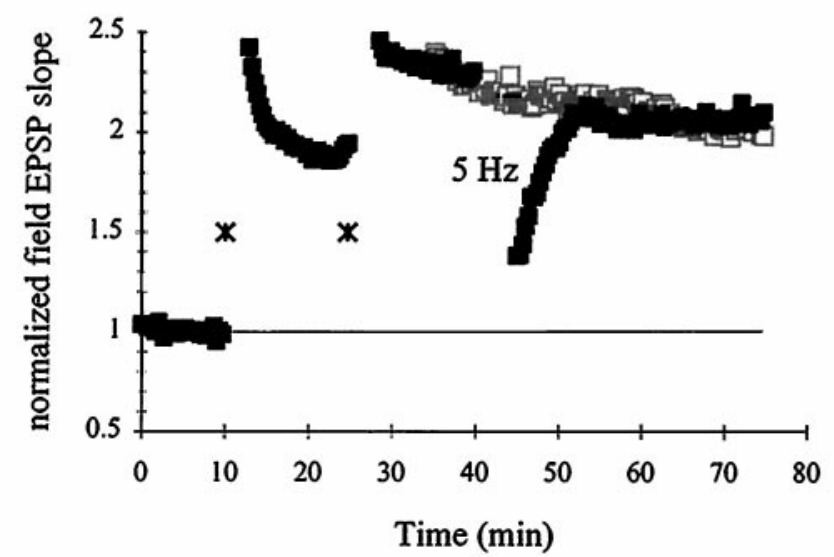

$\mathrm{C}$

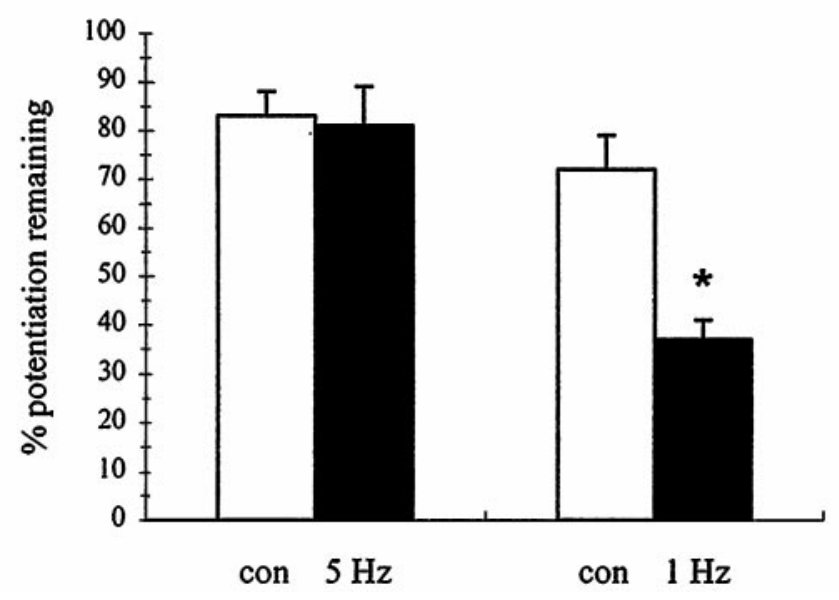

region of hippocampal slices obtained from young (2-3 weeks) rats. Namely, the depression is dependent on the activation of NMDA receptors and is homosynaptic. This demonstrates that the type of LTD induced by LFS can occur throughout the developmental life of the animal when the conditions present during LTD induction are appropriate. Several recent reports have found that LFS-induced LTD can occur in the mature animal (Heynen et al., 1996; Manahan-Vaughan, 1997) or does not occur in the mature animal (Errington et al., 1995; Staubli and Scafidi, 1997). We would suggest that issues related to the presence or absence of priming conditions in a particular experimental preparation may contribute to the disparity among these studies.

In addition to bidirectional modification of synaptic strength, there is a growing recognition of the existence of modifications of synaptic plasticity based on previous activation of the synapse (Christie and Abraham, 1992; Huang et al., 1992; Wexler and Stanton, 1993). Because the effects of subsequent plasticityinducing protocols can be altered without an observable effect on the baseline synaptic strength, a genuine plasticity of synaptic plasticity (i.e., metaplasticity) has seemingly occurred. The first apparent characteristic of our form of metaplasticity (referred to as priming in our study) is that NMDA receptor activation was
B

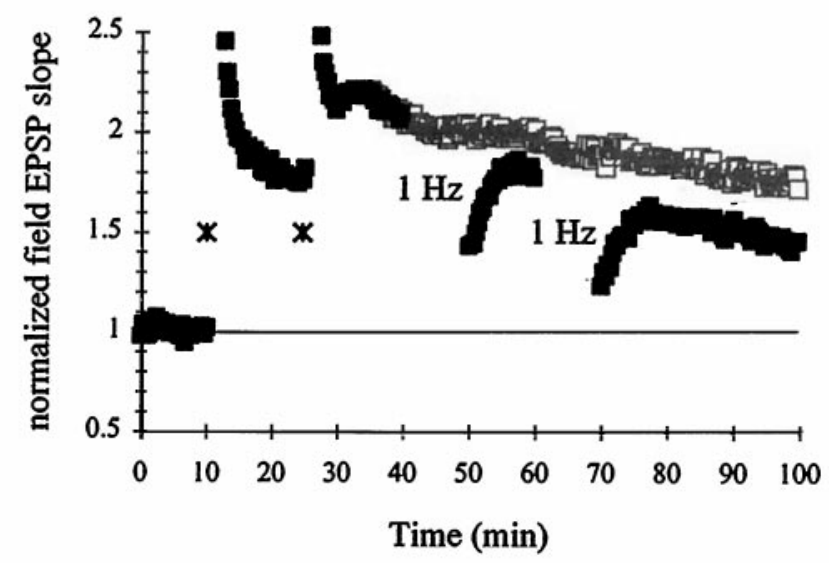

Figure 5. The LTP resulting from a multiple-tetanus induction protocol is differentially sensitive to depotentiation. $A$, A summary plot $(n=6)$ of the LTP produced in one pathway after multiple-tetanus HFS (asterisks, i.e., the priming protocol). At 15 min after priming, a $5 \mathrm{~Hz}$ depotentiation protocol consisting of two 1 min episodes separated by $2.5 \mathrm{~min}$ was performed (solid squares). Superimposed is the time course of a control, multiple-tetanus group of experiments $(n=$ 10 ), which did not experience $5 \mathrm{~Hz}$ stimulation (open squares). These control data were renormalized to the $5 \mathrm{~min}$ period immediately preceding $5 \mathrm{~Hz}$ stimulation to account for a small difference in the magnitude of LTP in the two groups. $B$, A summary plot $(n=4)$ of the LTP produced in one pathway after multiple-tetanus HFS (asterisks). Beginning $15 \mathrm{~min}$ after HFS, a $1 \mathrm{~Hz}$ depotentiation protocol (two episodes of 600 pulses) was performed (solid squares). Superimposed is the time course of a control group $(n=8)$, which also had experienced heterosynaptic priming, but not $1 \mathrm{~Hz}$ stimulation (open squares). As in $A$, these control data were renormalized to the $5 \mathrm{~min}$ period immediately preceding $1 \mathrm{~Hz}$ stimulation to account for a small difference in the magnitude of LTP in the two groups. $C$, Summary quantitation from the experiments above. Error bars are the mean \pm SEM, and the asterisk indicates a significant difference compared with the control pathway condition ( $p<0.01$, unpaired $t$ test).

not required during HFS protocol (Wagner and Alger, 1995). We also investigated two additional fundamental properties of this priming protocol: the duration of the priming effect and its pathway specificity. A significant enhancement of LTD lasted for at least $1.5 \mathrm{hr}$, but $<2.5 \mathrm{hr}$. The potential significance of this temporal window is discussed below. Interestingly, the effect of priming was heterosynaptic, particularly when priming was performed in the absence of APV. Subsequent induction of LTD was facilitated in either the primed pathway or in the unprimed, independent pathway. This suggests that this form of priming is being manifested at synapses throughout the postsynaptic cell. Because homosynaptic priming, as well as some heterosynaptic priming (albeit inconsistent), occurs in the presence of APV, we propose that the increased efficacy of heterosynaptic priming in the absence of APV is attributable to an enhancement of the spread of the priming effects when NMDA receptors can be activated, rather than suggest an a priori requirement for NMDA receptor activation in the priming of heterosynaptic pathways. In any case, it is presumed that the absence of APV would more closely approach the in vivo condition.

With respect to potential mechanisms of priming, several postsynaptic possibilities have been proposed (Gold and Bear, 1994; Stanton, 1995) and discussed recently (Bear, 1995; Abra- 
ham, 1996). These possibilities generally involve alterations in the handling of intracellular $\mathrm{Ca}^{2+}$ levels as a trigger for calciumdependent processes that then underlie metaplasticity. In addition to these direct, postsynaptic mechanisms, it is perhaps worth noting that a form of LTD has been described recently in stratum radiatum interneurons of the CA1 region that is NMDA receptor-independent, heterosynaptic, and induced by a multipletetanus protocol similar to our priming protocol (McMahon and Kauer, 1997). The indirect effect of decreased inhibitory tone after priming (i.e., disinhibition) could represent an important contributory step leading to pyramidal cell excitation and enhanced postsynaptic $\mathrm{Ca}^{2+}$ levels. The extensive connective network between relatively few interneurons and many pyramidal cells in the CA1 region (for review, see Freund and Buzsaki, 1996) would ensure a widespread (and possibly heterosynaptic) effect of any such disinhibition. A finding potentially related to this issue is that LTD evoked by LFS in vivo has been found to be dependent on the specific synaptic input being activated such that LFS of the ipsilateral Schaffer inputs, but not LFS of contralateral commissural inputs, results in LTD (Heynen et al., 1996). Because these two types of synaptic stimulation are known to activate different inhibitory circuits in the CA1 region (Andersen et al., 1964; Alger and Nicoll, 1979), a role for GABAergic systems in modulating LTD induction is implied.

Multiple-tetanus HFS protocols, such as those used in our priming studies, have been shown to elicit a protein synthesisdependent form of LTP, or late LTP (Frey et al., 1988; Otani et al., 1989; Huang and Kandel, 1994). It has been suggested recently that late LTP induction in a given pathway initiates a stabilization process that can spread throughout the postsynaptic neuron and stabilize the potentiation of less potent LTP induction protocols at other synaptic inputs. For a period of at least 40 min after the completion of tetanization, a weaker tetanus given at an independent pathway can "commandeer" stabilization factors produced previously, such that it too becomes late LTP (Frey and Morris, 1997). This heterosynaptic stabilization could be inappropriate in certain cases and raises the question as to how the neuron might regulate such occurrences. Because the same HFS conditions causing late LTP in the studies cited above also facilitate LTD induction in our preparation, we examined the role priming may have as a safeguard mechanism by acting to limit the inappropriate stabilization of LTP. Our results demonstrate that late LTP induction in one pathway produces an increased effectiveness of both the baseline depression caused by $1 \mathrm{~Hz}$ stimulation as well as the depotentiating effects of $5 \mathrm{~Hz}$ stimulation in another, independent pathway (i.e., heterosynaptic priming of LTD and depotentiation). These findings represent at least two possible ways by which inappropriate stabilization of weak potentiation could be avoided. First, the facilitation of LFS-induced LTD (Fig. 3) could have an inhibitory effect on the single-tetanus induction of LTP via the $100 \mathrm{~Hz} / 1$ sec protocol. Such an effect possibly is related to reports that previous synaptic activation via $1 \mathrm{~Hz}$ stimulation suppresses LTP induction (Fujii et al., 1991, 1996). An enhancement of this suppressive effect would tend to prevent the initiation of potentiation in other pathways by weak induction protocols. Second, the facilitation of $5 \mathrm{~Hz}$-induced depotentiation (Fig. 4) could provide a means by which weak potentiation could be reversed before it was stabilized. Increasing the susceptibility of weak potentiation to theta rhythm-induced depotentiation would tend to erase inappropriate potentiation of other pathways. Priming also may increase the time during which the weak potentiation is susceptible to reversal by theta-like patterns of stimulation (a similar effect has been reported to result during application of a drug that facilitates glutamate receptor activity) (Staubli and Chun, 1996). An important feature of these potential safeguards is that they are not likely to affect true late LTP induction and expression in the second pathway, because strong LTP induction usually is not blocked by previous $1 \mathrm{~Hz}$ stimulation (Wagner and Alger, 1995), and our results in Figure 5 show that the maintenance of late LTP is not affected significantly by $5 \mathrm{~Hz}$ depotentiation. These differential effects then allow for the preservation of the original late LTP and the induction and maintenance of late LTP in independent pathways while enhancing LTD and depotentiation in weakly potentiated pathways.

In summary, our results indicate that priming resulting from multiple HFS trains can have several implications for future occurrences of synaptic plasticity. When considering the potential significance of a given metaplasticity episode, it is important to appreciate that the resulting modulatory effects will be different depending on the type of subsequently evoked plasticity event. For example, a priming episode can potentially inhibit an attempt to potentiate the synapse (Huang et al., 1992) but also can facilitate depression of the synapse after similar priming stimuli (Wexler and Stanton, 1993). In both of these instances, a general inhibitory environment is promoted, via either suppressed potentiation or enhanced depression. We have found that our more intense priming protocol can facilitate the effectiveness of depotentiation by $5 \mathrm{~Hz}$ stimulation in a heterosynaptic pathway, whereas Frey and Morris (1997) have found that such an intense stimulation pattern can promote the stabilization of weak potentiation in a heterosynaptic pathway. Thus, both negative and positive influences on LTP stabilization can be present after the same priming event. In this report, we have shown that the facilitation of LTD induction in the primed pathway and the facilitation of LTD and depotentiation in the unprimed pathway can all occur after HFS priming, in addition to the immediate homosynaptic LTP produced. This multiplicity provides a stiff challenge for future attempts to unravel the significance of these interactions.

\section{REFERENCES}

Abraham WC (1996) Activity-dependent regulation of synaptic plasticity (metaplasticity) in the hippocampus. In: The hippocampus: functions and clinical relevance (Kato N, ed), pp 15-26. Amsterdam: Elsevier.

Abraham WC, Bear MF (1996) Metaplasticity: the plasticity of synaptic plasticity. Trends Neurosci 19:126-130.

Alger BE, Nicoll RA (1979) GABA-mediated biphasic inhibitory responses in hippocampus. Nature 281:315-317.

Andersen P, Eccles JC, Loyning YJ (1964) Location of synaptic inhibitory synapses on hippocampal pyramids. J Neurophysiol 27:592-607.

Bear MF (1995) Mechanism for a sliding synaptic modification threshold. Neuron 15:1-4.

Bienenstock EL, Cooper LN, Munro PW (1982) Theory for the development of neuron selectivity: orientation specificity and binocular interaction in visual cortex. J Neurosci 2:32-48.

Christie BR, Abraham WC (1992) Priming of associative long-term depression in the dentate gyrus by $\theta$ frequency synaptic activity. Neuron 9:79-84.

Dudek SM, Bear MF (1992) Homosynaptic long-term depression in area CA1 of hippocampus and effects of $N$-methyl-D-aspartate receptor blockade. Proc Natl Acad Sci USA 89:4363-4367.

Errington ML, Bliss TVP, Richter-Levin G, Yenk K, Doyere V, Laroche S (1995) Stimulation at 1-5 Hz does not produce long-term depression or depotentiation in the hippocampus of the adult rat in vivo. J Neurophysiol 74:1793-1799.

Frey U, Morris GM (1997) Synaptic tagging and long-term potentiation. Nature 385:533-536. 
Frey U, Krug M, Reymann KG, Matthies H (1988) Anisomycin, an inhibitor of protein synthesis, blocks late phases of LTP phenomena in the hippocampal CA1 region in vitro. Brain Res 452:57-65.

Frey U, Huang YY, Kandel ER (1993) Effects of cAMP simulate a late stage of LTP in hippocampal CA1 neurons. Science 260:1661-1664.

Freund TF, Buzsaki G (1996) Interneurons of the hippocampus. Hippocampus 6:347-470.

Fujii S, Saito K, Miyakawa H, Ito K, Kato H (1991) Reversal of longterm potentiation (depotentiation) induced by tetanus stimulation of the input to CA1 neurons of guinea pig hippocampal slices. Brain Res 555:112-122.

Fujii S, Kuroda Y, Miura M, Furuse H, Sasaki H, Kaneko K, Ito K, Chen Z, Kato H (1996) The long-term suppressive effect of prior activation of synaptic inputs by low-frequency stimulation on induction of longterm potentiation in CA1 neurons of guinea pig hippocampal slices. Exp Brain Res 111:305-312.

Gold JI, Bear MF (1994) A model of dendritic spine $\mathrm{Ca}^{2+}$ concentration exploring possible bases for a sliding synaptic modification threshold. Proc Natl Acad Sci USA 91:3941-3945.

Hebb DO (1949) The organization of behavior. New York: Wiley.

Heynen AJ, Abraham WC, Bear MF (1996) Bidirectional modification of CA1 synapses in the adult hippocampus in vivo. Nature 381:163-166.

Huang Y-Y, Kandel ER (1994) Recruitment of long-lasting and protein kinase A-dependent long-term potentiation in the CA1 region of hippocampus requires repeated tetanization. Learn Mem 1:74-82.

Huang Y-Y, Colino A, Selig DK, Malenka RC (1992) The influence of prior synaptic activity on the induction of long-term potentiation. Science 255:730-733.

Manahan-Vaughan D (1997) Group 1 and 2 metabotropic glutamate receptors play differential roles in hippocampal long-term depression and long-term potentiation in freely moving rats. J Neurosci 17:3303-3311.

McMahon LL, Kauer JA (1997) Hippocampal interneurons express a novel form of synaptic plasticity. Neuron 18:295-305.

Mulkey RM, Malenka RC (1992) Mechanisms underlying induction of homosynaptic long-term depression in area CA1 of the hippocampus. Neuron 9:967-975.

Otani S, Marshall CJ, Tate WP, Goddard GV, Abraham WC (1989) Maintenance of long-term potentiation in rat dentate gyrus requires protein synthesis but not messenger RNA synthesis immediately posttetanization. Neuroscience 28:519-526.

Sejnowski TJ (1977) Storing covariance with nonlinearly interacting neurons. J Math Biol 4:303-321.

Stanton PK (1995) Transient protein kinase C activation primes longterm depression and suppresses long-term potentiation of synaptic transmission. Proc Natl Acad Sci USA 92:1724-1728.

Staubli U, Chun D (1996) Factors regulating the reversibility of longterm potentiation. J Neurosci 16:853-860.

Staubli U, Scafidi J (1997) Studies on long-term depression in area CA1 of the anesthetized and freely moving rat. J Neurosci 17:4820-4828.

Wagner JJ, Alger BE (1995) GABAergic and developmental influences on homosynaptic LTD and depotentiation in rat hippocampus. J Neurosci 15:1577-1586.

Wagner JJ, Alger BE (1996) Homosynaptic LTD and depotentiation: do they differ in name only? Hippocampus 6:24-29.

Wexler EM, Stanton PK (1993) Priming of homosynaptic long-term depression in hippocampus by previous synaptic activity. NeuroReport 4:591-594. 